\title{
Mesenteric mass in a 28-year-old woman
}

\author{
Amitabh Gautam, Ajay K Khatri, Manoj Pandey, Nakul C Arya, Vijay K Shukla
}

A 28-year-old woman presented with a painless lower abdominal mass for six months. There was no history of previous pregnancy or abdominal surgery. On examination there was a mobile lump in the umbilical region which was nontender and firm with a smooth surface. Haematological and biochemical investigations were within normal limits. Ultrasonography revealed a homogenous solid mass without pressure effects. On exploratory laparotomy through a midline incision a whitish 5-cm round tumour was found in the mesentery of the ileum (figure 1). The mass did not extend up to the serosal surface of the bowel and it was possible to completely excise it.

The cut surface was homogenous, grey/white and had a fibrous look. A photomicrograph of a section from the mass is shown in figure 2 .

\author{
Institute of Medical \\ Sciences, Banaras \\ Hindu University, \\ Varanasi 221 005, India \\ Department of \\ Surgery \\ A Gautam \\ AK Khatri \\ $M$ Pandey \\ VK Shukla \\ Department of \\ Pathology
}

NC Arya

Correspondence to Dr VK Shukla

Accepted 12 December 1995

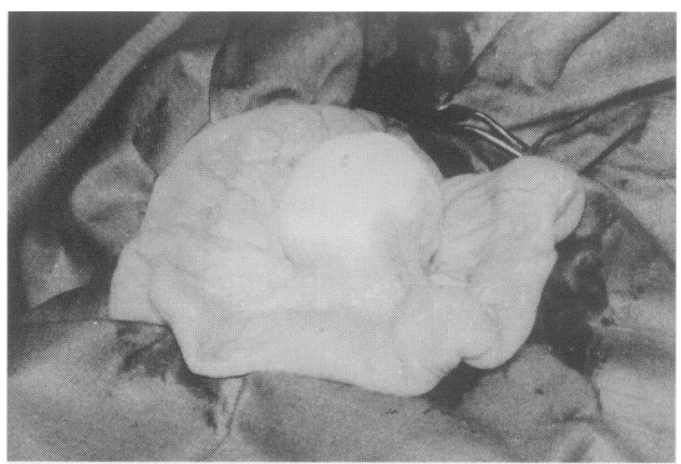

Figure 1 The excised tumour

\section{Question}

What is this condition called?

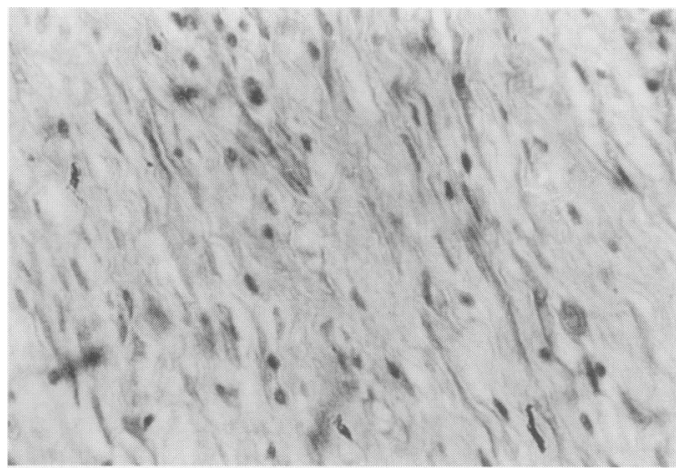

Figure 2 Section from tumour (H \& E stain, original $\times 500$ ) 
Table Reported cases of primary mesenteric fibromatosis (from ${ }^{4}$ )

\begin{tabular}{lllll}
\hline Ref & Age & Sex & Treatment & Follow-up \\
\hline 1 & 27 & M & complete excision & ? \\
2 & 34 & M & incomplete excision & recurrent disease; died after 3.5 years \\
4 & 22 & M & complete excision & recurrent disease; died after 6 months \\
Hailemarien, cited in 4 & 37 & M & complete excision & 8 months; no recurrence \\
Pilegard, cited in 4 & 42 & M & incomplete excision & no recurrence \\
present case & 28 & F & complete excision & 3 years; no recurrence \\
\hline
\end{tabular}

\section{Answer}

Primary mesenteric fibromatosis. Histological examination revealed a spindle cell tumour of moderate cellularity without atypia, composed of broad bundles and sheets of parallel oriented fibroblasts separated by dense fibrillary collagen (figure 2). The margins of resection were clear. There was no family history of polyposis and sigmoidoscopic examination showed no abnormalities. The patient has been under follow-up for three years, with clinical and repeated ultrasonological examinations, without any evidence of recurrence.

\section{Discussion}

Mesenteric fibromatosis, also referred to as 'desmoid tumour' of the mesentery, is a rare, histologically benign but locally aggressive non-metastasizing tumour. It is notorious for local recurrence if excision is incomplete. ${ }^{1}$ It commonly occurs in association with familial adenomatous polyposis, especially if Gardner's syndrome is present. ${ }^{1-3}$ The other predisposing causes reported are pregnancy and abdominal surgery or trauma. ${ }^{4}$ Mesenteric fibromatosis occurring in the absence of any of the known predisposing factors is rare. David and Khanduri ${ }^{4}$ called it primary mesenteric fibromatosis and reported five cases from the literature (table). All these patients were males. Mesen-

1 Forte MD, Brant WE. Spontaneous isolated mesenteric fibromatosis. Dis Colon Rectum 1988; 31: 315-7.

2 Kinn AC, Haggmark T, Willems JS. Aggressive mesenteric fibromatosis. Acta Chir Scand 1989; 155: 293-6.

3 Lotfi AM, Dozois RR. Mesenteric fibromatosis complicating familial adenomatous polyposis - predisposing factors and results of treatment. Int $f$ Colorectal Dis $1989 ; 4: 30-6$.

4 David SS, Khanduri P. A case of primary mesenteric David SS, Khanduri P. A case of primary
fibromatosis. Aust $N \mathcal{F}$ Surg 1992; 62: 813-4.

5 Tsukada K, Church JM, Jagelman DG, et al. Noncytotoxic drug therapy for intra abdominal desmoid tumour in patients with familial adenomatous polypuses. Dis Colon Rectum 1992; 35: 29-33. teric fibromatosis, in general, is more common in women of child-bearing age, as hormonal influence is strongly implicated ${ }^{4}$ and yet ours is the first female case of primary mesenteric fibromatosis. It presents as a mass in the abdomen which is usually asymptomatic, ${ }^{2}$ leading to delayed presentation. Thus, in two of the five previously reported patients, the disease had already spread to retroperitoneal structures. ${ }^{2,4}$ Surgery is the mainstay of treatment and complete excision of the tumour is curative. Inadequate excision results in recurrance within a year as observed in two cases. Both radiotherapy and chemotherapy (cytotoxic and non-cytotoxic) have been used for recurrent and inoperable disease in mesenteric fibromatosis with variable results. ${ }^{5}$ Non-cytotoxic drug therapy with nonsteroidal antiinflammatory drugs (sulindac and indomethacin) and anti-oestrogenic drugs (tamoxifen) has been reported to be effective in obtaining remission in some cases. ${ }^{5-8}$ These drugs may therefore be useful in cases of primary mesenteric fibromatosis with residual disease after excision.

\section{Final diagnosis}

Primary mesenteric fibromatosis.

Keywords: mesenteric fibromatosis, desmoid tumour

6 Kinzbrunner B, Ritter S, Domingo J, Rosenthal J. Remission of rapidly growing desmoid tumours after tamoxifen therapy. Cancer 1983; 52: 2201-4.

$7 \mathrm{Kim} \mathrm{DH}$, Goldsmith HS, Quam SH, Huvos AG. Intraabdominal desmoid tumour. Cancer 1971; 27: 1041-5.

8 Waddel WK, Gerner RE. Indomethacin and ascorbate inhibits desmoid tumours. F Surg Oncol 1980; 15: 85-90. 